\title{
Wide-area Detection of Voltage Instability from Synchronized Phasor Measurements. Part I: Principle
}

\author{
Mevludin Glavic, Senior Member, IEEE
}

\author{
Thierry Van Cutsem, Fellow, IEEE
}

\begin{abstract}
This two-part paper deals with the early detection of an impending voltage instability from the system states provided by synchronized phasor measurements. Recognizing that voltage instability detection requires assessing a multi-dimensional system, the method fits a set of algebraic equations to the sampled states, and performs an efficient sensitivity computation in order to identify when a combination of load powers has passed through a maximum. The important effects of overexcitation limiters are accounted for. The approach does not require any load model. This first part of the paper is devoted to theoretical foundations of sensitivity calculation along the system trajectory, derivation of the algebraic model, and illustration on a simple 5-bus system involving the long-term dynamics of a load tap changer and a field current limiter.
\end{abstract}

Index Terms-Long-term voltage stability, instability detection, wide-area monitoring, phasor measurement units, sensitivity analysis

\section{INTRODUCTION}

$\mathbf{T}$ He phasor measurement technology [1], [2], developed since the end of the 80's, together with advances in computational facilities, networking infrastructure and communications, have opened new perspectives for designing widearea monitoring, detection, protection and control systems. The Phasor Measurement Unit (PMU) hardware is now based on proven technology and is considered as the most accurate and advanced time-synchronized technology available to power engineers [3].

As documented in several comprehensive surveys [3], [4], [5], present and potential applications of synchronized phasor measurements range from mere monitoring to tracking system dynamics in real-time. The study reported in this paper belongs to the last category, as we assume that the monitored region is equipped with PMUs ensuring full observability of bus voltages within that region. Admittedly, present-day power systems are still far from having such a rich measurement configuration. However, it is likely that in some future all measurement devices will be provided with high precision time tags [3]. Furthermore, incentive to invest in such a rich measurement configuration will be driven by the preliminary demonstration of its potential benefits in monitoring, protection and control functions.

M. Glavic (glavic@montefiore.ulg.ac.be) is visiting professor, supported by an FNRS (Fund for Scientific Research) grant, at the Dept. of Electrical Engineering and Computer Science of the University of Liège, Sart Tilman B37, B-4000 Liège, Belgium.

T. Van Cutsem (t.vancutsem@ulg.ac.be) is a research director of FNRS and adjunct professor at the same department.
Voltage stability has been identified as one area where those PMU-enhanced functions could prove useful to prevent system blackouts and the associated social and economical losses.

There are two complementary lines of defence against voltage instability [6], [7]: preventive and corrective. As regards preventive aspects, PMUs can help improving the quality of present-day state estimation so that better initial operating points are available for real-time voltage security assessment applications [7], [8]. They can also improve modelling accuracy [3]. However, the corrective (or emergency) line of defence is where the PMU technology are likely to help most significantly. In this respect, this paper explores how it could help early detecting an impending long-term voltage instability, driven typically by Load Tap Changers (LTCs), OverExcitation Limiters (OELs) and restorative loads [6], [7].

Present-day System Integrity Protection Schemes (SIPS) against voltage instability mostly rely on the detection of low voltage conditions, possibly complemented by signals such as excessive field currents in neighouring generators [9]. The undervoltage criterion allows simple and possibly distributed SIPS, for instance for load shedding [10]. However, it essentially relies on the observation of already degraded operating conditions. The challenge is thus to demonstrate that PMU-based approaches can offer better anticipation capabilities by detecting the inception of instability rather than its consequences.

PMU-based voltage instability monitoring can be classified into two broad categories:

1) methods based on local measurements, with few or no information exchange between the monitoring locations. Most of them rely on Thévenin impedance matching condition [11], [12] or its extensions [13], [14], [15]. As long as the various buses are checked independently of each other, these methods accommodate the time skew of SCADA data and no time synchronization is needed;

2) methods requiring the observability of the whole region prone to voltage instability [16], [17], [18], [19]. They offer the potential advantages of wide-area monitoring. The measurements should be time-synchronized in so far as an accurate, dynamic tracking of system states is sought. They should preferably be filtered by a (linear) state estimator.

As already mentioned, the approach of this paper belongs to the second category. Comparisons with Thévenin impedance matching are offered in the companion paper [20]. 
Long-term voltage instability can be triggered by transmission and/or generation outages or by severe load increases. Although most of incidents experienced so far were triggered by outages, a large part of the existing literature concentrates on smooth load increase scenarios. On the contrary, this work focuses on detecting the onset of voltage instability triggered by a large disturbance. This requires accounting for the "noise" introduced on phasor measurements by short-dynamics not directly linked to long-term voltage instability.

Simply stated, voltage instability is linked to the inability of the combined generation-transmission system to provide the power requested by loads, as a result of equipment outages and limitations of reactive power generation [7]. In this perspective, we propose to compute sensitivities around the "snapshot" system states computed from PMUs, with the purpose of detecting that some combination of load powers has gone through a maximum.

The use of sensitivities in voltage stability analysis is not new [8], [21], [22], [23], [24]. Eigenvalue or singular value analysis of various Jacobian matrices has been also proposed for quite some time (e.g. [25], [26], [27]). Initially intended to provide preventive security indices [28], these linearization-based techniques have been superseded by methods that account for system nonlinearities (such as load power margin computation). Nevertheless, they may prove useful in scrutinizing the unstable system behaviour triggered by a large disturbance. These aspects have been comparatively less investigated, with the exception of [27] where eigenvalues are computed at selected snapshots of the unstable system evolution and [23], [24] where sensitivity analysis is coupled to a simplified time-domain simulation. Sensitivity of dynamic system responses have been studied through trajectory sensitivities (e.g. [29]). The latter are computationally much more demanding than the sensitivity of (pseudo-)equilibria considered in this work. Also, it is not clear to the authors whether the many discrete controls and delays present for instance in tap changers, switched capacitors, random load switching, etc. can be easily taken into account.

In this work, it was chosen not to rely on a dynamic model to predict the system response (e.g. [14], [30]). Indeed, this requires a reliable model, especially for loads in emergency conditions. Also, it is not clear how to reconcile the model with the measured system evolution, in case of discrepancies caused, for instance, by events not accounted for in the model. The proposed approach does not try to anticipate the load response, but it anticipates generator limitations.

In principle the computations presented in this paper could rely on bus voltages provided by a standard state estimator processing SCADA measurements. However, SCADA data are not collected and state estimators are not run at the rate considered here (and hence some proposed filtering would not be possible). Furthermore, standard (nonlinear) state estimators may encounter convergence problems in degraded system conditions. Last but not least, the data collected by RTUs suffer from time skew that could make the proposed computations unreliable in the presence of significant transients.

This paper does not consider the important problems of PMU placement, communication infrastructure, measurement pre-processing by a state estimator, etc. Instead, we simply assume that PMUs provide synchronized bus voltage phasors. In our simulations, they are obtained from detailed timedomain simulation. Circuit breaker statuses are supposed to be provided by the same equipment or by the SCADA system.

The paper is organized as follows. In Section II, the theoretical background of sensitivity analysis is reviewed and extended to tracking eigenvalue movement around a maximum load power point. The system equilibrium model fitted to each snapshot is detailed in Section III. The various assumptions and techniques are illustrated on a simple system in Section IV, while concluding remarks are offered in Section V.

Notation. Lowercase bold letters indicate column vectors. Uppercase bold letters refer to matrices. ${ }^{T}$ denotes transposition. Complex numbers are overlined.

\section{THEORETICAL BACKGROUND}

\section{A. System trajectory}

Let $\mathbf{s}$ be the vector of active and reactive powers consumed by the loads. Thus, in a system with $N_{l}$ load buses, the dimension of $\mathbf{s}$ is $n_{s}=2 N_{l}$. Let the system be characterized by a state vector $\mathbf{z}$ of dimension $n_{z}$. In response to a disturbance, both $\mathbf{s}$ and $\mathbf{z}$ evolve with time. To this evolution corresponds a trajectory in the $\left(n_{s}+n_{z}\right)$-dimensional space of the $\left[\mathbf{z}^{T} \mathbf{s}^{T}\right]^{T}$ vectors.

We assume that this trajectory obeys:

$$
\phi(\mathbf{z}, \mathbf{s})=\mathbf{0}
$$

where $\phi: R^{n_{s}+n_{z}} \rightarrow R^{n_{z}}$ is assumed to be a smooth function. The next section will be devoted to deriving a practical set of equations of this type. Let us already stress that the proposed method amounts to assessing the above model linearized around sampled points of the trajectory obtained from synchronized phasor measurements.

A two-dimensional picture of such a trajectory is sketched in Fig. 1, where $n_{s}=n_{z}=1$. As both $\mathbf{z}$ and $\mathbf{s}$ evolve with time, the system operating point moves along the curve as suggested by the arrows.

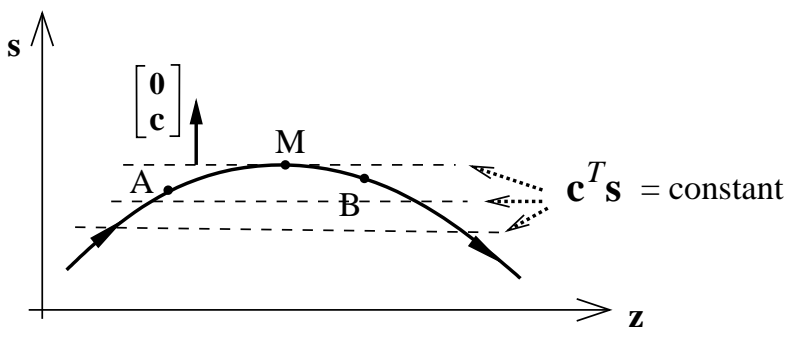

Fig. 1. Two-dimensional illustration of system trajectory

Our goal is to identify one point of the trajectory where some linear combination $f=\mathbf{c}^{T} \mathbf{s}$ of the load powers passes through a maximum, where $\mathbf{c}$ is an $n_{s}$-dimensional nonzero vector. Note that $\mathbf{c}$ is not known beforehand. In the simple case of Fig. 1 with $n_{s}=1$, the $f$ function amounts to $f=c \times s$. The equi- $f$ curves are the dotted lines parallel to the $z$ axis. In the general case, the equi- $f$ locus is an hyperplane orthogonal to the vector $\left[\mathbf{0}^{T} \mathbf{c}^{T}\right]^{T}$. The maximum value of $f$ is reached 
at point $\mathrm{M}$, where one of the equi- $f$ line is tangent to the trajectory.

\section{B. Property of point $M$}

Let us show that the Jacobian of $\phi$ with respect to $\mathbf{z}$ is singular at point $\mathrm{M}$.

Indeed, this point is a solution of the constrained optimization problem:

$$
\begin{array}{cc}
\max _{\mathbf{s}, \mathbf{z}} & f=\mathbf{c}^{T} \mathbf{s} \\
\text { subject to } & \phi(\mathbf{z}, \mathbf{s})=\mathbf{0}
\end{array}
$$

The Karush-Kuhn-Tucker necessary optimality conditions of the above problem are obtained by setting to zero the derivatives of the Lagrangian:

$$
\mathcal{L}=\mathbf{c}^{T} \mathbf{s}+\mathbf{w}^{T} \phi(\mathbf{z}, \mathbf{s})
$$

with respect to $\mathbf{z}, \mathbf{s}$ and $\mathbf{w}$. This yields:

$$
\begin{aligned}
\phi(\mathbf{z}, \mathbf{s}) & =\mathbf{0} \\
\phi_{\mathbf{z}}^{T} \mathbf{w} & =\mathbf{0} \\
\mathbf{c}+\phi_{\mathbf{s}}^{T} \mathbf{w} & =\mathbf{0}
\end{aligned}
$$

where $\phi_{\mathrm{z}}$ (resp. $\phi_{\mathrm{s}}$ ) denotes the Jacobian of $\phi$ with respect to $\mathbf{z}$ (resp. $\mathbf{s}$ ). Since $\mathbf{c}$ is nonzero, Eq. (6) implies that $\mathbf{w}$ is also nonzero. Hence, from (5), one easily concludes that the Jacobian $\phi_{\mathrm{z}}$ is singular. Equivalently, $\phi_{\mathrm{z}}$ has a zero eigenvalue; $\mathbf{w}$ is the corresponding left eigenvector.

As is well known, second-order conditions have to be met in order the solution of (4-6) to be a (local) maximum. We assume that those conditions hold in the situations of concern here, where loads tend to increase their powers over the period of time that follows a disturbance.

Conversely, at a point where $\phi_{\mathrm{z}}$ is singular, Eq. (5) holds with a nonzero vector $\mathbf{w}$. If this vector is such that $\phi_{\mathrm{s}}^{T} \mathbf{w}=\mathbf{0}$, no combination of load powers is found to reach an extremum. In the more general case where $\phi_{\mathrm{s}}^{T} \mathbf{w} \neq \mathbf{0}$, the point can in principle be interpreted as a local extremum of a combination of load powers. However, this may not correspond to voltage instability. In practice, singular Jacobians are not expected in normal operating conditions but may be observed in degraded conditions.

\section{Eigenvalue movement along the trajectory}

We show next that when passing through $\mathrm{M}$, one real eigenvalue of $\phi_{\mathrm{z}}$ changes sign.

Let us consider the "speed" vector $\left[\begin{array}{ll}\dot{\mathbf{z}}^{T} & \dot{\mathbf{s}}^{T}\end{array}\right]^{T}$, where the dots denote time derivative. Since this vector is tangent to the trajectory, we have:

$$
\left[\begin{array}{ll}
\phi_{\mathrm{z}} & \phi_{\mathrm{s}}
\end{array}\right]\left[\begin{array}{c}
\dot{\mathrm{z}} \\
\dot{\mathrm{s}}
\end{array}\right]=\mathbf{0}
$$

from which one easily derives:

$$
\dot{\mathrm{z}}=-\phi_{\mathrm{z}}^{-1} \phi_{\mathrm{s}} \dot{\mathrm{s}}
$$

Let us assume that $\phi_{\mathbf{z}}$ has all distinct eigenvalues ${ }^{1}$ so that its inverse can be decomposed into:

$$
\phi_{\mathbf{z}}^{-1}=\sum_{i=1}^{n_{z}} \frac{\mathbf{v}_{i} \mathbf{w}_{i}^{T}}{\lambda_{i}}
$$

where $\mathbf{v}_{i}$ and $\mathbf{w}_{i}$ are the right and left eigenvectors relative to the eigenvalue $\lambda_{i}$, respectively. In the neighbourhood of point $\mathrm{M}$, one eigenvalue - say $\lambda_{c}$ - is close to zero. Hence, the term relative to $\lambda_{c}$ dominates in (9) and the following approximation holds:

$$
\phi_{\mathbf{z}}^{-1} \simeq \frac{\mathbf{v}_{c} \mathbf{w}_{c}^{T}}{\lambda_{c}}
$$

Introducing (10) into (8) yields:

$$
\dot{\mathbf{z}} \simeq-\frac{\mathbf{v}_{c} \mathbf{w}_{c}^{T}}{\lambda_{c}} \phi_{\mathrm{s}} \dot{\mathbf{s}}
$$

Furthermore, in the neighbourhood of $\mathbf{M}, \mathbf{w}_{c}$ is expected to be very close to the left eigenvector of the zero eigenvalue, which satisfies (6). Using the latter equation, (11) can be rewritten as:

$$
\dot{\mathbf{z}} \simeq \mathbf{v}_{c} \frac{\mathbf{c}^{T} \dot{\mathbf{s}}}{\lambda_{c}}
$$

In this last expression, $\mathbf{c}^{T} \dot{\mathbf{s}}$ represents the time derivative of $f=\mathbf{c}^{T} \mathbf{s}$. Coming back to Fig. 1, consider point $\mathrm{A}$ of the trajectory, close to $\mathrm{M}$ but reached before $\mathrm{M}$. At this point, the time derivative of $f$ is positive. Similarly, at point B close to $\mathrm{M}$ but reached after $\mathrm{M}$, the time derivative of $f$ is negative.

Assuming that the trajectory passes smoothly from A to B through M, the speed vector $\dot{z}$ is continuous. Hence, in (12) the change in sign of $\mathbf{c}^{T} \dot{\mathbf{s}}$ must be compensated by the opposite change in sign of $\lambda_{c}$. In other words, as the system passes from $\mathrm{A}$ to $\mathrm{B}$, one real eigenvalue $\lambda_{c}$ changes sign.

\section{Using sensitivities}

To detect the change in sign of one real eigenvalue, there is no need to explicitly compute eigenvalues of $\phi_{\mathbf{z}}$.Instead, sensitivities involving the inverse Jacobian $\phi_{\mathbf{z}}^{-1}$ can be used.

We consider the sensitivities of the total reactive power generation to individual load reactive powers. Let the load reactive powers be grouped into $\mathbf{q}=\left[\begin{array}{lll}Q_{1} & \ldots & Q_{N_{l}}\end{array}\right]^{T}$. The sought sensitivities are obtained from a general sensitivity formula [7] as:

$$
S_{Q_{g} \mathbf{q}}=-\phi_{\mathbf{q}}^{T}\left(\phi_{\mathbf{z}}^{T}\right)^{-1} \nabla_{\mathbf{z}} Q_{g}
$$

where $\nabla_{\mathbf{z}} Q_{g}$ denotes the gradient of $Q_{g}$ with respect to $\mathbf{z}$ and the $\phi_{\mathbf{q}}$ is the Jacobian of $\phi$ with respect to $q$. With the model detailed in the next section, this matrix includes 0's and 1's.

In normal operating conditions, the above sensitivities are positive, and usually larger than one. As point $\mathrm{M}$ is approached, one real eigenvalue $\lambda_{c}$ approaches zero and the sensitivities increase. After crossing point $\mathrm{M}$, the sensitivities are negative due to the change in sign of $\lambda_{c}$. As the trajectory

\footnotetext{
${ }^{1}$ We exclude the case of two (or more) zero eigenvalues as it corresponds to two (or more) distinct combinations of load powers being maximum at the same time.
} 
leaves point $\mathrm{M}, \lambda_{c}$ moves away from zero and the sensitivities decrease in magnitude. Thus, when passing through $\mathrm{M}$, the sensitivities change sign through infinity.

In theory, all sensitivities $S_{Q_{g} Q_{j}}$ change sign at the same time, whatever the bus $j$. In practice, however, this effect is less pronounced as one moves away from the region experiencing the largest voltage drops, because the numerator in (10) becomes smaller [24].

In practice, discontinuities and trajectory sampling may prevent sensitivities from reaching very high values, as will be illustrated later on. What is sought is a sudden change in sign, i.e. we seek to identify a discrete time $k$ such that:

$$
S_{Q_{g} Q_{j}}(k-1)>d_{+} \quad \text { and } \quad S_{Q_{g} Q_{j}}(k)<d_{-}
$$

where $d_{+}>0$ and $d_{-}<0$ are thresholds to be adjusted.

Computing $S_{Q_{g} \mathbf{q}}$ merely requires solving one linear system with $\phi_{\mathbf{z}}$ as matrix of coefficients and $\nabla_{\mathbf{z}} Q_{g}$ as independent term. The main computational effort lies in the factorization of $\phi_{\mathbf{z}}$, for which efficient sparsity programming packages are available. In turn, the most expensive step is the optimal ordering of the matrix, which can be done only infrequently (after major changes in topology, otherwise zero entries can be used for outaged equipment). Sparse vector techniques could take advantage of the zero components of $\nabla_{\mathbf{z}} Q_{g}$. Finally, the Jacobian can be limited to the region prone to voltage instability, as shown in the companion paper [20].

Eigenvalue computation is comparatively more demanding because it requires solving iteratively a sequence of linear systems of the same size. Furthermore, tracking the movement of eigenvalues in case of discontinuities due to e.g. OELs adds some complexity. For detection purposes, the easily computed sensitivities were found to work satisfactorily.

\section{SYSTEM MODELLING}

\section{A. Basic assumptions}

The following basic assumptions are made:

- the network is represented by its standard bus admittance matrix. Real-time breaker status information is used to assemble this matrix;

- the short-term dynamics of generators, automatic voltage regulators, speed governors, static var compensators, etc. are not tracked but replaced by accurate equilibrium equations. This assumption is reasonable in so far as longterm voltage instability is of concern. Provision is made for large transients that cause the system to deviate from the assumed equilibrium;

- the long-term dynamics driven by OverExcitation Limiters (OELs), Load Tap Changers (LTCs) and restorative loads are reflected through the change in measured voltages from one snapshot to the next;

- whether a generator is voltage controlled or field current limited is known or detected. Equations are adjusted accordingly;

- since the method aims at detecting a maximum of a combination of load powers, only the consumed powers need to be known; no information about load behaviour is needed in the proposed method.

\section{B. Overview of the model}

Based on the above assumptions, the algebraic model (1) is obtained as follows.

Decomposing the bus admittance matrix $\overline{\mathbf{Y}}$, the vector $\overline{\mathbf{V}}$ of bus voltages and the vector $\overline{\mathbf{I}}$ of nodal currents into their real and imaginary parts:

$$
\overline{\mathbf{Y}}=\mathbf{G}+j \mathbf{B} \quad \overline{\mathbf{V}}=\mathbf{v}_{x}+j \mathbf{v}_{y} \quad \overline{\mathbf{I}}=\mathbf{i}_{x}+j \mathbf{i}_{y}
$$

the network relations $\overline{\mathbf{I}}=\overline{\mathbf{Y}} \overline{\mathbf{V}}$ take on the form:

$$
\begin{aligned}
& \mathbf{G} \mathbf{v}_{x}-\mathbf{B} \mathbf{v}_{y}-\mathbf{i}_{x}=\mathbf{0} \\
& \mathbf{B} \mathbf{v}_{x}+\mathbf{G v}_{y}-\mathbf{i}_{y}=\mathbf{0}
\end{aligned}
$$

We take the $r$-th bus as reference by setting the phase angle of its voltage to zero, or equivalently:

$$
v_{y r}=0
$$

The short-term dynamics model can be written in compact form as:

$$
\begin{aligned}
\dot{\mathbf{x}} & =\mathbf{f}\left(\mathbf{x}, \mathbf{v}_{x}, \mathbf{v}_{y}, \mathbf{i}_{x}, \mathbf{i}_{y}\right) \\
\mathbf{0} & =\mathbf{g}\left(\mathbf{x}, \mathbf{v}_{x}, \mathbf{v}_{y}, \mathbf{i}_{x}, \mathbf{i}_{y}\right)
\end{aligned}
$$

where $\mathbf{x}$ includes state variables such as flux linkages, rotor speeds, controls, etc. while (20) relates to the stator of synchronous and induction machines, static loads, SVCs and other FACTS devices, etc. Assuming short-term dynamics at equilibrium as mentioned above, (19) is replaced by:

$$
\mathbf{0}=\mathbf{f}\left(\mathbf{x}, \mathbf{v}_{x}, \mathbf{v}_{y}, \mathbf{i}_{x}, \mathbf{i}_{y}\right)
$$

The active and reactive powers consumed by the load at the $i$-th $\left(i=1, \ldots, N_{l}\right)$ bus relate to voltage and current components through:

$$
\begin{aligned}
& v_{x i} i_{x i}+v_{y i} i_{y i}+P_{i}=0 \\
& v_{y i} i_{x i}-v_{x i} i_{y i}+Q_{i}=0
\end{aligned}
$$

Hence, the algebraic model (1) consists of Eqs. (16, 17, 18, $20,21,22,23)$ involving the vectors of variables:

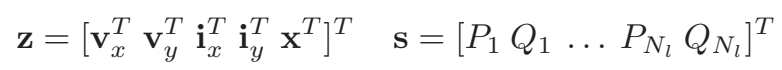

Finally, the total reactive power generation $Q_{g}$ used in (13) is given by:

$$
Q_{g}=\sum_{i=1}^{N_{r}}\left(v_{y i} i_{x i}-v_{x i} i_{y i}\right)
$$

where the sum extends over all generator, synchronous condenser and static compensator buses.

\section{Modelling the synchronous machine and its controls}

In this section, the synchronous generator model is considered in some detail. Other components are modelled in the same spirit.

In theory, Eqs. (21) are obtained by setting the left hand side of (19) to zero. In practice, however, the reduced model detailed hereafter, extensively used in Quasi Steady-State (QSS) simulation [7], [23], offers a good compromise between simplicity and accuracy. 
Each synchronous machine is characterized by the emf $\bar{E}_{q}$ whose magnitude is proportional to the field current, and the emf $\bar{E}_{q}^{s}$ behind saturated synchronous reactances. Assuming negligible armature resistance, the stator equations are written in the machine $(d, q)$ reference frame as:

$$
\left(\begin{array}{c}
0 \\
E_{q}^{s}
\end{array}\right)=\left(\begin{array}{c}
V_{d} \\
V_{q}
\end{array}\right)+\left(\begin{array}{cc}
0 & X_{q}^{s} \\
-X_{d}^{s} & 0
\end{array}\right)\left(\begin{array}{c}
I_{d} \\
I_{q}
\end{array}\right)
$$

where $X_{d}^{s}$ and $X_{q}^{s}$ are the saturated direct- and quadrature-axis synchronous reactances, respectively. In the system $(x, y)$ axes the above equation becomes:

$$
\begin{aligned}
& \left(\begin{array}{c}
0 \\
E_{q}^{s}
\end{array}\right)=\left(\begin{array}{cc}
-\sin \delta & \cos \delta \\
\cos \delta & \sin \delta
\end{array}\right)\left(\begin{array}{c}
v_{x} \\
v_{y}
\end{array}\right) \\
& +\left(\begin{array}{cc}
0 & X_{q}^{s} \\
-X_{d}^{s} & 0
\end{array}\right)\left(\begin{array}{cc}
-\sin \delta & \cos \delta \\
\cos \delta & \sin \delta
\end{array}\right)\left(\begin{array}{c}
i_{x} \\
i_{y}
\end{array}\right)
\end{aligned}
$$

where $\delta$ is the rotor angle, i.e. the phase angle of $\bar{E}_{q}^{s}$ in the $(x, y)$ reference frame. $X_{d}^{s}$ and $X_{q}^{s}$ relate to their unsaturated values $X_{d}$ and $X_{q}$ through:

$$
X_{d}^{s}=X_{\ell}+\frac{X_{d}-X_{\ell}}{k} \quad X_{q}^{s}=X_{\ell}+\frac{X_{q}-X_{\ell}}{k}
$$

where $X_{\ell}$ is the leakage reactance and

$$
k=\frac{E_{q}}{E_{q}^{s}}
$$

is the saturation coefficient. A widely used model is:

$$
k=1+m\left(V_{\ell}\right)^{n}
$$

where $V_{\ell}$ is the magnitude of the voltage behind leakage reactance, defined by:

$$
\bar{V}_{\ell}=\bar{V}+j X_{\ell} \bar{I}
$$

Expressing voltages and currents in terms of their components, (30) becomes:

$$
k-1-m\left[\left(v_{x}-X_{\ell} i_{y}\right)^{2}+\left(v_{y}+X_{\ell} i_{x}\right)^{2}\right]^{n / 2}=0
$$

With $X_{d}^{s}$ and $X_{q}^{s}$ given by (28), the two equations in (27) make up the stator equations of the type (20). There are three algebraic variables of the type $\mathbf{x}$, namely $E_{q}^{s}, \delta$ and $k$, that must be balanced by three equations of the type (21). The first of these equations is (32). The remaining two are provided by voltage and speed regulations, as detailed next.

For voltage control, one of the following relations holds:

- under Automatic Voltage Regulator (AVR) control:

$$
E_{q}=G\left(V^{o}-V\right) \Leftrightarrow k E_{q}^{s}-G\left(V^{o}-\sqrt{v_{x}^{2}+v_{y}^{2}}\right)=0
$$

where $G$ is the open-loop static gain and $V^{o}$ the voltage setpoint;

- under field current limit:

$$
E_{q}=E_{q}^{l i m} \Leftrightarrow k E_{q}^{s}-E_{q}^{l i m}=0
$$

These equations are easily extended to error-free voltage regulation, constant reactive power or armature current limit.

Speed control is accounted for by:

$$
P=P^{o}-\alpha \Delta f_{s} \Leftrightarrow v_{x} i_{x}+v_{y} i_{y}-P^{o}+\alpha \Delta f_{s}=0
$$

where $P^{o}$ is the power setpoint, $\alpha$ involves the permanent speed droop and $\Delta f_{s}$ is the deviation of the system frequency with respect to its nominal value. This equation is easily modified in case the turbine operates at its limit.

With the short-term electromechanical oscillations neglected, $\Delta f_{s}$ is common to all generators. This additional variable is balanced by the phase reference equation (18).

\section{Fitting the $\mathbf{z}$ variables to real-time measurements}

As previously explained, at each time step, synchronized values of the bus voltage phasors $\hat{\mathbf{V}}$ are assumed to be received either from PMUs or (preferably) from a state estimator. The corresponding value of $\mathbf{z}$ is easily obtained as follows.

Referring all phasors to the $r$-th bus and decomposing $\hat{\mathbf{V}}$ into real and imaginary parts yields the voltages $\hat{\mathbf{v}}_{x}$ and $\hat{\mathbf{v}}_{y}$.

The corresponding nodal currents $\hat{\mathbf{i}}_{x}$ and $\hat{\mathbf{i}}_{y}$ are obtained from the network equations $(16,17)$.

Formally, the state $\mathbf{x}$ is obtained by solving (21) with $\mathbf{v}_{x}, \mathbf{v}_{y}, \mathbf{i}_{x}$ and $\mathbf{i}_{y}$ set at their values $\hat{\mathbf{v}}_{x}, \hat{\mathbf{v}}_{y}, \hat{\mathbf{i}}_{x}$ and $\hat{\mathbf{i}}_{y}$. For the previously detailed generator model, the procedure is as follows:

1) Obtain the active and reactive currents as:

$$
\begin{aligned}
& I_{P}=P / V=\left(v_{x} i_{x}+v_{y} i_{y}\right) / \sqrt{v_{x}^{2}+v_{y}^{2}} \\
& I_{Q}=Q / V=\left(v_{y} i_{x}-v_{x} i_{y}\right) / \sqrt{v_{x}^{2}+v_{y}^{2}}
\end{aligned}
$$

2) determine the saturation coefficient $k$ from (32) and the saturated reactances from (28)

3) determine the generator internal angle [7]:

$$
\varphi=\operatorname{arctg}\left(\frac{X_{q}^{s} I_{P}}{V+X_{q}^{s} I_{Q}}\right)
$$

4) and therefrom the rotor angle $\delta=\varphi+\operatorname{arctg}\left(v_{y} / v_{x}\right)$

5) compute $E_{q}^{s}=\left(V+X_{d}^{s} I_{Q}\right) \cos \varphi+X_{d}^{s} I_{P} \sin \varphi$

6) and therefrom the emf $E_{q}=k E_{q}^{s}$.

\section{E. Fitting the model to OEL status}

The switching of generators from AVR to OEL control plays an important role in voltage instability [6], [7] and, hence, it must be accounted for.

In the best case, information could be sent by power plants about the status of their field current: below limit (normal operating mode), over limit (temporary overexcitation), limited (after OEL activation).

To avoid sending the OEL status, $E_{q}$ can be compared to $E_{q}^{l i m}$ corresponding to the rotor current enforced by the OEL. If $E_{q}<E_{q}^{l i m}-\epsilon$, the machine is under AVR control and (33) is used. If $E_{q}>E_{q}^{l i m}+\epsilon$, it is concluded that the OEL is going to act after the overload time is elapsed. The tolerance $\epsilon$ accounts for noise, OEL inaccuracy, etc.

Furthermore, anticipation of the OEL activation will be considered in the companion paper [20]. 


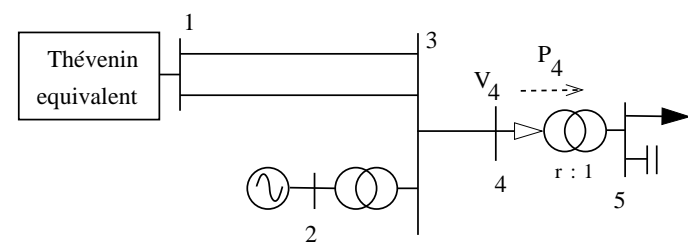

Fig. 2. 5-bus test system

\section{ILLUSTRATION OF METHOD ON A 5-BUS SYSTEM}

\section{A. System description}

The system, largely inspired of one detailed in [7], is shown in Fig. 2. It involves the Thévenin equivalent of a remote system, one generator, and one load fed through a transformer equipped with LTC. Most of the load power is provided by the remote system (bus 1) through a rather long, double-circuit transmission line.

The load behaves as constant current and the LTC has different delays on its first and subsequent tap changes. For the generator at bus 2, a 6th-order model is used, together with a simple representation of its speed governor, AVR and OEL. The OEL has an inverse-time characteristic.

The disturbance considered is the tripping of one circuit between buses 1 and 3 , at $t=1 \mathrm{~s}$. The system response is obtained by variable-step numerical integration. All bus voltage phasors are sampled every $0.1 \mathrm{~s}$, and used as measurements $\hat{\mathbf{V}}$ (without adding noise in this simple example). This sampling rate is higher than needed for tracking long-term voltage instability, but compatible with recent technology [1], [3]. It was chosen to track the effect of short-term transients.

\section{B. Case 1: LTC starts responding after OEL has acted}

We first consider a situation where the OEL is activated before the LTC starts responding, so that their effects are clearly separated in time.

The evolution of the voltage at bus 4 is shown with heavy line in Fig. 3. One can recognize the effect of:

- quickly damped initial electromechanical oscillations;

- the OEL acting at $t=30 \mathrm{~s}$, thereby withdrawing voltage support near the load;

- the LTC that starts acting at $t=51 \mathrm{~s}$, trying to restore the voltage at distribution bus 5 , and hence the load power;

- eventually, a faster instability causing voltage collapse.

As already mentioned, the sensitivities refer to a model that neglects short-term dynamics. To illustrate the accuracy of this simplification, Fig. 4 shows the difference between the emf $E_{q}$ provided by detailed time simulation and its approximation computed from snapshots, assuming the generator dynamics at equilibrium, as detailed in Section III-D. A discrepancy is observed essentially during the transients that follow the line tripping and, to a much lower extent, when the generator is switched under field current limit. The difference should be compared to the limit $E_{q}^{l i m}=2.825$ pu enforced by the OEL; it remains very small.

Expectedly, a larger error is experienced when an initial short-circuit (cleared by the same line opening) is considered,

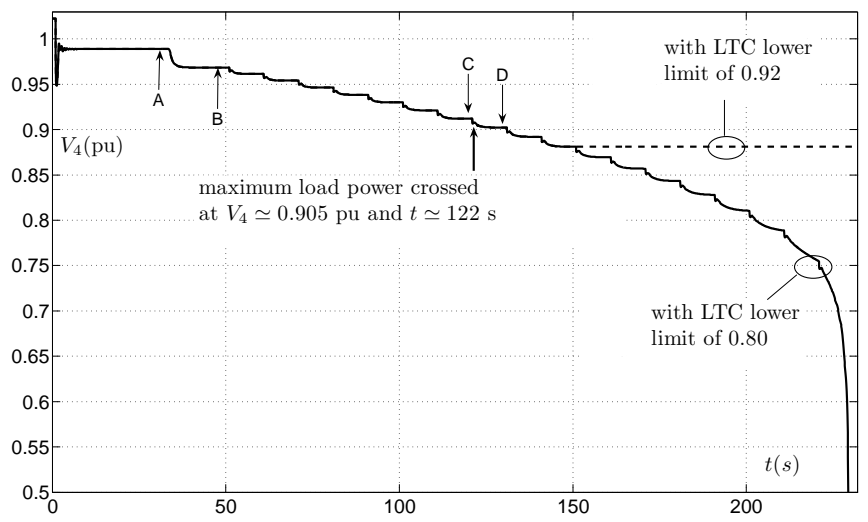

Fig. 3. Case 1: Voltage at bus 4

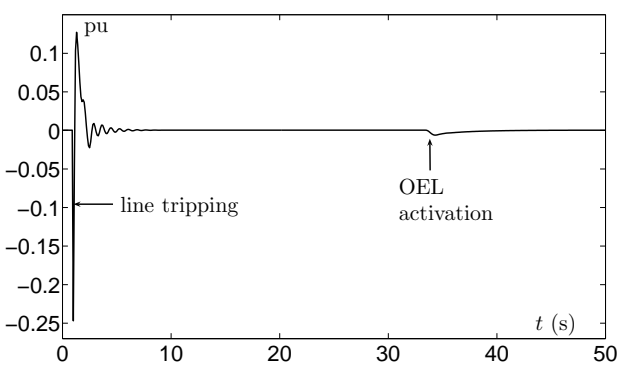

Fig. 4. Case 1: discrepancy between the $E_{q}$ values provided by detailed simulation and model at equilibrium, respectively

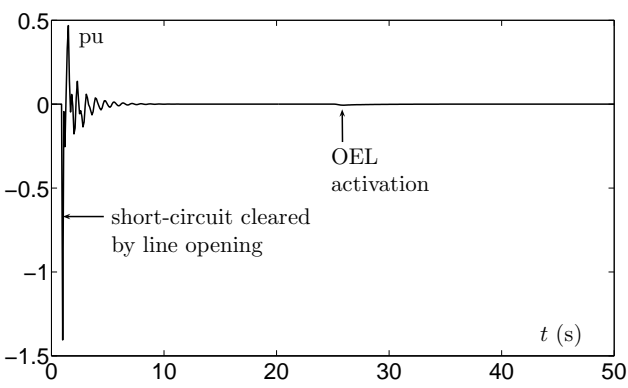

Fig. 5. Same discrepancy as in Fig. 4 but with an initial short-circuit applied

as shown in Fig. 5. However, the discrepancy does not last for a long time, and changes sign owing to the oscillatory response of the system. Such transients can be easily accounted for by waiting for some time (typically 1 second) before issuing an alarm.

Before and after the OEL action, as well as in between successive tap changes the system has time to reach ShortTerm Equilibrium (STE) points [7]. A, B, C and D in Fig. 3 are examples of such points. Each of them satisfies the algebraic model (1) (namely Eqs. 16, 17, 18, 20, 21, 22, 23) for different OEL and LTC states. These points are also shown in the next two figures.

In Fig. 6 voltage $V_{4}$ has been plotted as a function of load active power $P_{4}$ (see Fig. 2) in the range of values of interest. $\mathrm{O}$ is the initial operating point. The system PV curve after OEL action is shown with dashed line; it has been obtained by joining the STE points such as B, C, and D.

There from, the instability mechanism is easily seen. Both the line tripping and the field current reduction decrease the 


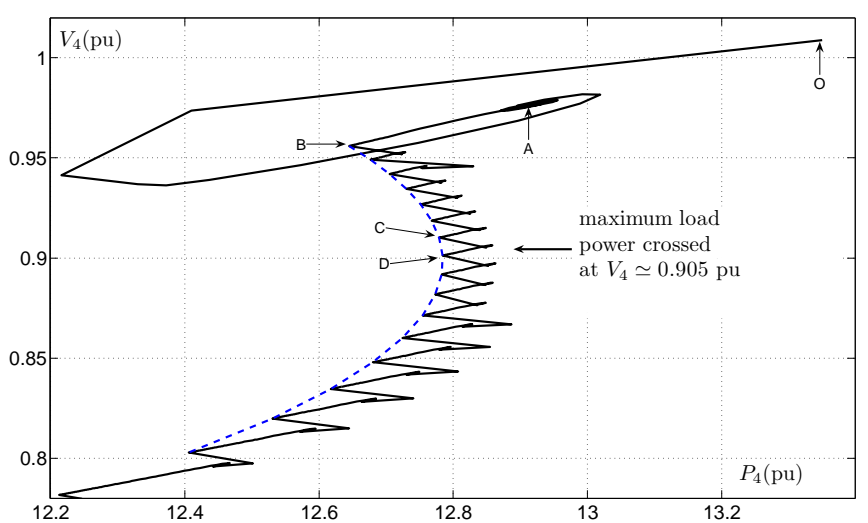

Fig. 6. Case 1: PV curve at bus 4

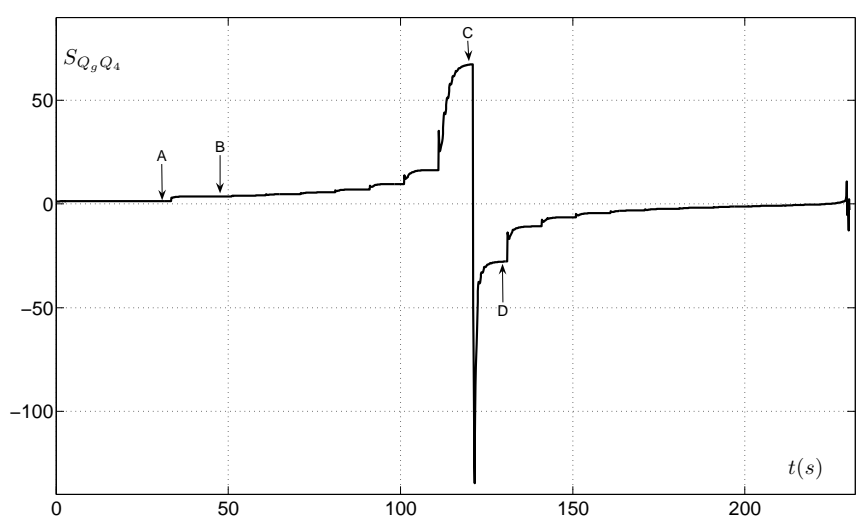

Fig. 7. Case 1: Sensitivity $S_{Q_{g} Q_{4}}$

maximum power that can be delivered to the load to $12.77 \mathrm{pu}$, i.e. less than the initial load power of 13.35 pu that the LTC tries to restore indirectly.

Under the effect of the tap changes, the system passes through the maximum load power at a voltage $V_{4} \simeq 0.905 \mathrm{pu}$. It is seen from Fig. 3 that this takes place at $t \simeq 122 \mathrm{~s}$.

Figure 7 shows the evolution of the sensitivity $S_{Q_{g} Q_{4}}$. It represents the additional vars to be produced by the generator of bus 2 and the remote network when consuming 1 more var at bus 4 . The change in sign expected from theory is clearly seen. It takes place at $t \simeq 122 \mathrm{~s}$, which is in very good agreement with the curves in Figs. 3 and 6.

In principle, the sensitivity values are consistent with the assumption of steady-state operation only at the STE points. $\mathrm{C}$ is the last STE point where a positive sensitivity is obtained, while D the first where a negative value is observed. The sensitivity switches from +68 to -28 . In between two STE points, the system had some transients and the sensitivities at the sampled points took much larger values, as shown from the figure.

Further analysis shows that the Jacobian $\phi_{\mathbf{u}}$ has an eigenvalue $\lambda=-0.0054$ at point $\mathrm{C}$ and an eigenvalue $\lambda=+0.0091$ at point $\mathrm{D}$, thus confirming the zero crossing. From the corresponding (normalized) left eigenvector $\mathbf{w}$, the $\mathbf{c}$ vector is obtained from (6) as:

$$
\mathbf{c}=-\boldsymbol{\phi}_{\mathbf{s}}^{T} \mathbf{w}=\left[\begin{array}{cccc}
P_{3} & Q_{3} & P_{4} & Q_{4} \\
0.6199 & 0.9787 & 0.7341 & 1.0000
\end{array}\right]^{T}
$$

Note that bus 3 with its zero load has been treated as bus 4 . Thus, near the switching point, the linear combination of load powers $\mathbf{c}^{T} \mathbf{s}=0.6199 P_{3}+0.9787 Q_{3}+0.7341 P_{4}+Q_{4}$ is maximum.

Note that in standard load power margin computations the c vector is chosen beforehand, by specifying the pattern of load increase. Here, we have no control on c. It is dictated by the system dynamics, which are significant only in the area affected by the disturbance. The above values make sense, with components higher for reactive than active power, and at bus 4 , more remote from generation than bus 3 .

Note also that the $\mathbf{c}$ vector is not unique. Indeed, denoting by $\mathbf{s}_{O}$ any sub-vector of $\mathbf{s}$, and by $\mathbf{c}_{o}$ the corresponding sub-vector of $\mathbf{c}$, it is easily seen that $\mathbf{c}_{o}^{T} \mathbf{s}_{o}$ is maximum together with $\mathbf{c}^{T} \mathbf{s}$, provided the corresponding sub-vector of $\mathbf{w}$ is nonzero, and assuming that the other components of $\mathrm{s}$ are kept constant.

In this example, the instability of the LTC long-term dynamics is the trouble cause, and it results in instability of the short-term dynamics [7]. Indeed, in its unsuccessful attempt to restore the load voltage, the LTC drives the system to so low voltages that the field-current limited generator at bus 2 looses synchronism, and makes voltages plunge. This is easily checked by reducing the range of LTC action. The dotted curve in Fig. 3 corresponds to the lower limit of the ratio $r$ (see Fig. 2) increased from 0.80 (yielding the evolution shown with solid line) to 0.92 . In this case, the system does not reach the point where short-term dynamics becomes unstable, but settles at a voltage below $0.905 \mathrm{pu}$ corresponding to maximum power. The sensitivity evolves as in Fig. 7 up to $t=150 \mathrm{~s}$, from which it remains at a constant negative value.

As clearly shown by this example, the detection of maximum load power is an indication of long-term instability of LTCs, unable to restore load powers to their pre-disturbance values. Note that this detection takes place early before system collapse, when voltages are still "normal", typically higher than the setting of an undervoltage-based protection scheme.

It could be argued that the system evolution shown with dotted curve in Fig. 3 is not unstable. Indeed, the LTC hitting its limit has a stabilizing effect. On the other hand, the final operating point is little viable and certainly not secure. For instance, any increase in demand will cause further, uncontrollable transmission voltage drops. We thus believe the alarm issued by the sensitivity is acceptable in view of this and the early detection it offers in more dangerous scenarios.

Note finally that sensitivities change sign near the end of the simulation. This corresponds to the above mentioned loss of synchronism. This is of little concern since the system is already in obvious emergency condition.

\section{Case 2: LTC starts responding before OEL acts}

A shorter initial tap changer delay and a longer overload of the field current are now assumed, so that the former starts responding before the latter is activated. Furthermore, an initial operating point with $14.2 \mathrm{pu}$ of load active power is considered. The system response to the same disturbance is shown in Fig. 8. It is easily seen that the OEL acts after the 5th tap change. 


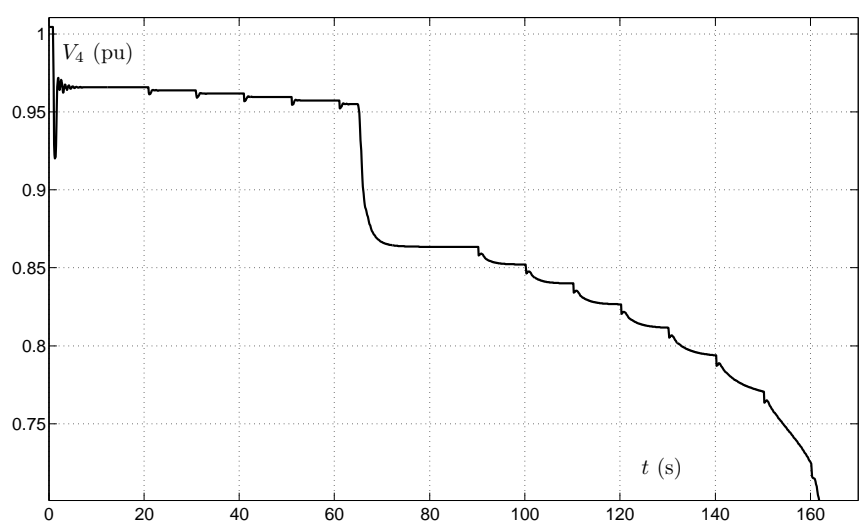

Fig. 8. Case 2 : Voltage of bus 4

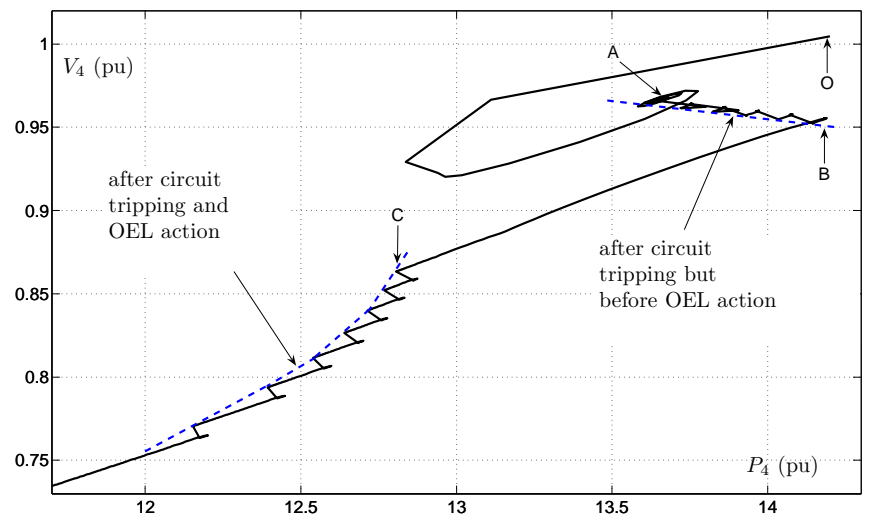

Fig. 9. Case 2: PV curves at bus 4

The PV curves obtained as in Case 1 are shown in Fig. 9. As a matter of fact, there is one PV curve before and one after the OEL activation. Note that the LTC had restored its voltage (and hence the load power) by the time the OEL acted (transition from B to C). Definitely, this is no longer possible after the field current is limited.

The interest of this example is in the fact that, when entering its final configuration, the system finds itself below the corresponding maximum power point, as suggested by the slope of the final PV curve near point C. Thus, the system does not cross a maximum power point but jumps from one side of a first maximum (point B) to the other side of a second maximum (point $\mathrm{C}$ ).

The sensitivity evolution is shown in Fig. 10. The last STE point with positive sensitivity is B while the first STE point with negative sensitivity is $\mathrm{C}$. As expected from the above remark, the sensitivities at point $\mathrm{B}(1.44)$ and at point $\mathrm{C}(-4.81)$ do not have as large magnitudes as in Case $1^{2}$. In between the two STE points, under the effect of transients, the sensitivities reach much larger values, emphasizing the change in sign. To face the case where no such transients would be observed, it is safe to take a lower $d_{+}$and/or a higher $d_{-}$in the test (14) in order to correctly identify the switching point.

\footnotetext{
${ }^{2}$ Sensitivities changing sign without passing through large (in theory infinite) values can be experienced under smooth parameter changes, when crossing a loadability limit where a generator reaches its limit [31], a case referred to as limit-induced bifurcation in [28]. In this example, however, the sensitivity jump results from the delayed operation of the OEL
}

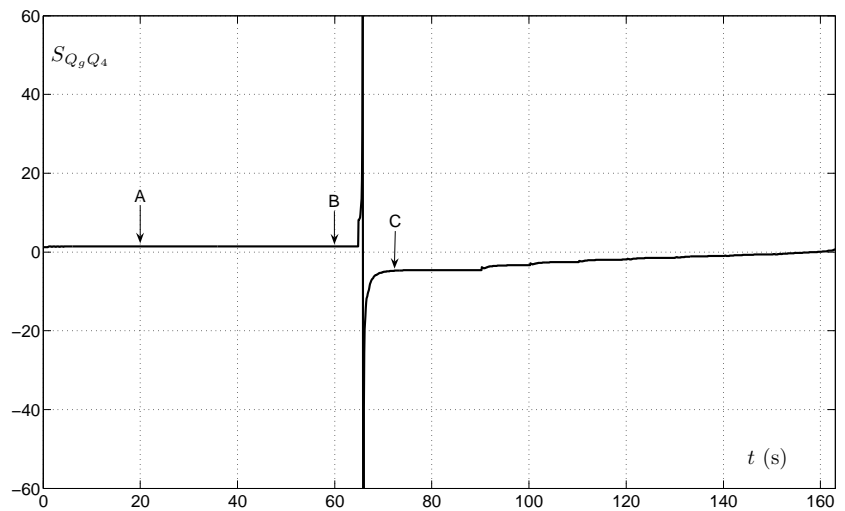

Fig. 10. Case 2: Sensitivity $S_{Q_{g} Q_{4}}$

As in Case 1, an early warning is obtained. An even earlier alarm can be obtained by anticipating the field current limitation of the generator, as shown in Part II [20].

\section{CONCLUSION}

This paper has revisited some properties of sensitivities and further investigated their behaviour in the neighbourhood of a maximum load power point, with the aim of devising a wide-area criterion to detect long-term voltage instability from bus voltage phasors provided by PMUs. The sensitivities of reactive power generation to reactive power loads are considered. Their change in sign through large values make up the sought criterion. The algebraic model from which the Jacobian is derived assumes the short-term dynamics to be at equilibrium. Load responses to disturbances do not appear in the model, thereby avoiding a great source of uncertainty in real-life applications. Last but not least, the sensitivity computation is quite compatible with the targeted real-time application.

The criterion has been illustrated on a 5-bus system. The criterion is shown to identify the point where the "nose" of the PV curve is crossed after a large disturbance leading to instability. This example also points out the sensitivity behaviour when an overexcitation limiter comes into play.

\section{REFERENCES}

[1] A. G. Phadke, "Synchronized Phasor Measurements in Power Systems," IEEE Computer Applications in Power, vol. 6, no. 2, pp. 10-15, 1993.

[2] A. G. Phadke, and J. S. Thorp, Synchronized Phasor Measurements and their Applicationa, Springer, 2008.

[3] D. Novosel, V. Madani, B. Bhargava, K. Vu, and J. Cole, "Dawn of the Grid Synchronization: Benefits, Practical Applications, and Deployment Strategies for Wide Area Monitoring, Protection, and Control," IEEE Power and Energy Magazine, vol. 6, no. 1, pp. 49-60, Jan. 2008.

[4] P. Pourbeik and C. Rehtanz (conveners), "Wide area monitoring and control for transmission capability enhancement," Final Report of CIGRE Working Group C4.601, Jan. 2007.

[5] A. G. Phadke, H. Voslkis, R. M. de Morales, T. Bi, R. N. Nayak, Y. K. Sehgal, S. Sen, W. Sattinger, E. Martinez, O. Samuelsson, D. Novosel, V. Madani, and Y. A. Kulikov "The Wide World of Wide-Area Measurements," IEEE Power and Energy Magazine, vol. 6, no. 5, pp.5265, Nov. 2008.

[6] C. W. Taylor, Power System Voltage Stability, EPRI Power System Engineering Series, McGraw Hill, 1994.

[7] T. Van Cutsem, C. Vournas, Voltage Stability of Electric Power Systems, Springer (previously Kluwer Academic Publishers), 1998. 
[8] V. Ajjarapu, Computational Techniques for Voltage Stability Assessment and Control, Springer, 2006

[9] D. H. Karlsson (convener), System Protection Schemes in Power Networks, Final report of CIGRE Task Force 38.02.19, Jun. 2001.

[10] B. Otomega and T. Van Cutsem, "Undervoltage load shedding using distributed controllers," IEEE Trans. Power Syst., vol. 22, no. 4, pp. 18981907, Nov. 2007.

[11] K. Vu, M. M. Begovic, D. Novosel, and M. M. Saha, "Use of Local Measurements to Estimate Voltage Stability Margin," IEEE Trans. on Power Syst., vol. 14, no. 3, pp. 1029-1035, Aug. 1999.

[12] S. Corsi and G. N. Taranto, "A Real-Time Voltage Instability Identification Algorithm Based on Local Phasor Measurements," IEEE Trans. on Power Syst., vol. 23, no. 3, pp. 1271-1279, Aug. 2008

[13] L. Warland, A. T. Holen, "Estimation of Distance to Voltage Collapse: Testing and Algorithm Based on Local Measurements", Proc. 14th Power System Computation Conference (PSCC), Paper s38p03, Sevilla (Spain), Jun. 2002

[14] C. Rehtanz and J. Bertsch, "Wide Area Measurement and Protection System for Emergency Voltage Stability Control," in Proc. 2002 IEEE Power Eng. Soc. Winter Meeting, New York, NY, Jan. 2002, pp. 842-847

[15] I. Smon, G. Verbic, and F. Gubina, "Local Voltage-Stability Index Using Tellegen's Theorem," IEEE Trans. on Power Syst., vol. 21, no. 3, pp. 1267-1275, Aug. 2006.

[16] B. Milosevic and M. Begovic, "Voltage Stability Protection and Control using a Wide-Area Network of Phasor Measurements," IEEE Trans. on Power Syst., vol. 18, no. 1, pp. 121-127, Feb. 2003.

[17] B. Genet and J-C. Maun, "Voltage Stability Monitoring using WideArea Measurement System," in Proc. IEEE Power Tech Conf., Lausanne, Switzerland, paper 392, Jun. 2007

[18] V. Balamourougan, T. S. Sidhu, and M. S. Sachdev, "Technique for online prediction of voltage collapse," IEE Proc. on Generation, Transmission and Distribution, vol. 151, no. 4, pp. 453-460, Jul. 2004

[19] M. Glavic and T. Van Cutsem, "Detecting with PMUs the onset of voltage instability caused by a large disturbance," in Proc. 2008 IEEE Power Eng. Soc. General Meeting, Pittsburgh, PA, Jul. 2008

[20] M. Glavic and T. Van Cutsem, "Wide-area detection of voltage instability from synchronized phasor measurements. Part II: Simulation results," companion paper submitted for publication in IEEE Trans. on Power Syst.
[21] J. Carpentier, R. Girard, and E. Scano, "Voltage collapse proximity indicators computed from an optimal power flow," in Proc. 1984 8th Power System Computation Conference (PSCC), Helsinki, Finland, 1984, pp. 671-678.

[22] M. Begovic and A. G. Phadke, "Control of voltage stability using sensitivity analysis," IEEE Trans. on Power Syst., vol. 7, no 1, pp. 114123, Feb. 1992.

[23] T. Van Cutsem, "An approach to corrective control of voltage instability using simulation and sensitivity," IEEE Trans. on Power Syst., vol. 10, no. 2, pp. 616-622, May 1995.

[24] F. Capitanescu and T. Van Cutsem, "Unified sensitivity analysis of unstable or low voltages caused by load increases or contingencies, "IEEE Trans. on Power Syst., vol. 20, no. 1, pp. 321-329, Feb. 2005.

[25] R. A. Schlueter, I. P. Hu, and T. Y. Guo, "Dynamic/static voltage stability security criteria," in Proc. 1991 Bulk Power Syst. Voltage Phenomena-II: Voltage Stability and Security, Deep Creek Lake (USA), Aug. 1991, pp. 265-310.

[26] P.-A. Löf, T. Smed, G. Andersson, and D.J. Hill, "Fast calculation of a voltage stability index," IEEE Trans. on Power Syst., vol. 7, no 1, pp. 54-64, Feb. 1992

[27] G. K. Morison, B. Gao, and P. Kundur, "Voltage Stability Analysis Using Static and Dynamic Approaches," IEEE Trans. on Power Syst., vol. 8, no. 3, pp. 1159-1171, Aug. 1993.

[28] C. Canizares (Editor/Coordinator), Voltage Stability Assessment: Concepts, Practices, and Tools, IEEE PES publication, Power System Stability Subcommittee, ISBN 0780378695, 2002.

[29] I. A. Hiskens and M. A. Pai, "Trajectory Sensitivity Analysis of Hybrid Systems," IEEE Trans. on Circuits and Systems-1: Fundamental Theory and Applications, vol. 47, no. 2, pp. 204-220, Feb. 2000.

[30] M. Zima and G. Andersson, "Model Predictive Control of Electric Power Systems under Emergency Conditions," in Real Time Stability in Power Systems: Techniques for Early Detection of the Risk of Blackout, S. C. Savulescu (editor), Springer, 2005.

[31] I. Dobson and L. Lu, "Voltage Collapse Precipitated by the Immediate Change in Stability When Generator Reactive Power Limits are Encountered," IEEE Trans. on Circuits and Systems-1: Fundamental Theory and Applications, vol. 39, no. 9, pp. 762-766, Sep. 1992.

Authors' biographies are available in the companion paper [20]. 